\title{
The Peripheral Blood Expression of Lipoic Acid Synthetase Gene as an Early Marker for Diabetic Nephropathy \\ Marwa M. Esawy ${ }^{1 *}$, Mahmoud M. Magdy ${ }^{2}$ \\ 1. Clinical Pathology Department, 2. Internal Medicine Department, Faculty of Medicine, Zagazig University, Egypt \\ *Corresponding Author: Marwa M. Esawy, Telephone Number: 00201066343326, Email: dr.marwaesawy@ymail.com,ORCID: 0000-0002-2198-258X
}

\begin{abstract}
Introduction: Diabetic nephropathy (DN) is one of the diabetic complications, which leads to end-stage renal disease. This study aimed to assess the expression of the lipoic acid synthetase (LIAS) gene in type 2 diabetic patients and DN patients.

Subjects and Methods: A case-control study conducted on 60 patients who had type 2 diabetes, 60 patients who had DN, and 60 healthy matched individuals. The peripheral blood expression of the LIAS gene was assessed by real-time qRT-PCR.

Results: In diabetic and DN patients, the expressions of LIAS were significantly lower than controls $(\mathrm{p}<0.001)$. The LIAS expression showed a reducing trend with the progress of DN $(\mathrm{p}<0.001)$. The LIAS expression showed a sensitivity of $95 \%$ and specificity of $88.3 \%$ in the differentiating between diabetic and DN patients and it can detect early DN with a sensitivity of $93.5 \%$ and specificity of $90 \%$. LIAS expression in DN patients had significant negative correlations with disease duration and albuminuria. LIAS gene expression can protect significantly from the DN. It had an odds ratio of 0.01 [95\% confidence interval (CI): 0.001-0.03] ( $\mathrm{p}<0.001)$. LIAS gene expression can significantly predict disease severity.

Conclusion: Peripheral blood expression of the LIAS gene was significantly lower in diabetic and DN patients in comparison to controls. The LIAS expression negatively correlated with the progress of DN. LIAS gene expression seems to be a promising marker for prediction and early detection of DN in type 2 diabetic patients.
\end{abstract}

Keywords: Diabetic nephropathy; Gene Expression; Lipoic acid synthetase.

\section{INTRODUCTION}

Lipoic acid synthetase (LIAS) gene was identified at human chromosome 4 in the p14 band ${ }^{(\mathbf{1})}$. The LIAS gene product is one of the mitochondrial proteins that is capable of alpha-lipoic acid synthesis ${ }^{(2)}$. Alpha-lipoic acid shows an obvious antioxidant activity ${ }^{(3)}$. Besides, it has a metal-chelating activity and reactive oxygen species-quenching activity that share in other natural antioxidants reduction ${ }^{(4)}$.

Diabetic nephropathy (DN) is a classic kidney abnormality in diabetic patients ${ }^{(5)}$. The oxidative stress is the main suspected cause of its development ${ }^{(6,7)}$. So, many studies directed toward the impact of supplementary antioxidant drugs in diabetes (8-10). Especial attention was given to the roles of alpha-lipoic acid in diabetes; it shares in the glycemic control and complications management ${ }^{(11)}$.

Alpha-lipoic acid can prevent $\mathrm{DN}$ by reducing malondialdehyde levels and restoring glutathione. These changes prevent basement membrane thickening, mesangial expansion, glomerulosclerosis, and fibrosis (12). Also, alpha-lipoic acid attenuates the DN manifestation either in animal models ${ }^{(\mathbf{1 3 - 2 0 )}}$ or humans ${ }^{(\mathbf{2 1} \text {, }}$ 22). So, studies were directed toward the LIAS gene and its expression in DN induced animal models ${ }^{(23,24)}$.

This study hypothesizes that LIAS gene expressions is reduced in diabetes and DN patients. This study aimed to evaluate the peripheral blood LIAS gene expression and its potential role as a new biomarker for $\mathrm{DN}$.

\section{SUBJECTS AND METHODS \\ Study design:}

A case-control design was utilized. This study was taken place in the Internal Medicine Department and the Clinical Pathology Department, Faculty of Medicine, Zagazig University. Over a 1 year period, between November 2017 and November 2018, this study was conducted.

\section{Ethical approval:}

This study protocol was approved by the Faculty of Medicine Institutional Review Board (Zagazig University). Before subjects' enrollment, all patients agreed and signed this study consent form.

\section{Subjects:}

The sample size was calculated based on a statistical power of 0.8 and a $95 \%$ confidence interval $(\mathrm{CI})$ to detect an assumed 0.7-fold difference in the gene expression. The equal allocation ratio for the study groups was utilized. This calculation was performed by the Epi Info program 6 (Atlanta, Georgia, USA). This study was conducted on 60 diabetic patients, $60 \mathrm{DN}$ patients, and 60 healthy matched individuals. 
Diabetes is diagnosed according to the American Diabetes Association criteria based on the fasting glucose concentration ${ }^{(25)}$. The DN diagnosis is based on the evidence of proteinuria of more than $300 \mathrm{mg}$ per day in a diabetic patient (26). Patients were excluded if they had infectious or hepatic diseases. Also, patients with renal diseases rather than DN were excluded.

\section{Sampling:}

All subjects were informed to fast for 12 hours. After 8 hours of fasting, whole blood was collected in one plain tube and two EDTA tubes (BD Vacutainer ${ }^{\circledR}$, Becton Dickinson \& $\mathrm{Co}, \mathrm{NJ}$ ). The plain tube was used to assess fasting glucose and kidney functions. The first EDTA tube was used for the Hemoglobin A1c (HbAlc) assay, and the second tube was utilized for the gene expression analysis. After another 4 hours of fasting, another sample was collected in a plain tube for the lipid profile assessment. A spot urine sample of 3-5 mL was collected from each subject.

After collection of the plain tubes, they were allowed to clot for 30 minutes at room temperature and after that were centrifuged at $1200 \mathrm{x}$ g for 10 minutes to separate serum.

\section{Method:}

Laboratory investigations were performed, including fasting blood glucose, HbAlc, cholesterol, triglycerides, high-density lipoprotein cholesterol (HDLC), and low-density lipoprotein cholesterol (LDL-C). Regarding the kidney function, it was assessed by measuring the serum creatinine, urinary creatinine, and urinary albumin; all laboratory tests were performed on Cobas 8000-C702 Modular Analyzer (Roche, Germany), except HbA1c performed on Cobas 6000C501 Modular Analyzer (Roche, Germany). The urinary albumin-creatinine ratio (UACR) was calculated by dividing the albumin concentration ( $\mathrm{mg}$ ) by the creatinine concentration (gm).

Within 1 hour of EDTA sample collection, the total RNA was extracted from the whole blood. RNA extraction was done according to the protocol of the used kits [Total RNA Purification kit, PP-210S] (Jena Bioscience, Germany). The quality of the extracted RNA was assessed using Nanodrop 2000 spectrophotometer (Thermo Fisher Scientific, Waltham, MA, USA). The integrity of the extracted RNA integrity was assessed by examining the ribosomal RNA bands gel electrophoresis.

The reverse transcription was performed by the SCRIPT Reverse Transcriptase kit [PCR-505S] (Jena Bioscience, Germany) with Oligo-(dT) primer. On the ice, $10 \mu \mathrm{L}$ of the extracted RNA, $1.5 \mu \mathrm{L}$ of RNase-free water, and $1 \mu \mathrm{L}$ of the primer were mixed. Then $4 \mu \mathrm{L}$ of the reverse transcription buffer, $1 \mu \mathrm{L}$ of dNTP Mix, $1 \mu \mathrm{l}$ of the RNase inhibitor, $1 \mu \mathrm{L}$ of the Dithiothreitol stock solution, and $0.5 \mu \mathrm{L}$ of the reverse transcriptase were added, mixed, and incubated at $28^{\circ} \mathrm{C}$ for $10 \mathrm{~min}$ and $50^{\circ} \mathrm{C}$ for $60 \mathrm{~min}$. The thermocycler [Gene Amp, PCR system 9700] was used for the reverse transcription (Perkin Elmer, Singapore).

The cDNA was preserved at $-80^{\circ} \mathrm{C}$ until used.

The primers were purchased from Metabion International AG (Planegg, Munich, Germany). The sequences of the primers were as the following:

LIAS gene (Forward primer: 5' TTCGTGAGGCAGATGTAGAC-3' and reverse primer: 5'- TATATGAAGAACGCACCAAAGG-3'), and RPS18 gene (Forward primer: 5'TAGCCTTTGCCATCACTGCC-3' and reverse primer: 5'-CATGAGCATATCTTCGGCCC-3').

Real-time qRT-PCR was carried out using the qPCR GreenMaster [PCR-313S] (Jena Bioscience, Germany). Twenty $\mu$ PCR reaction mix was prepared by adding 10 $\mu \mathrm{L}$ of Green Master, $0.5 \mu \mathrm{L}$ of the forward primer, $0.5 \mu \mathrm{L}$ of the reverse primer, $5 \mu \mathrm{L}$ of the cDNA, and $4 \mu \mathrm{L}$ of the PCR grade water. The initial denature was done at $95^{\circ} \mathrm{C}$ for 10 minutes, followed by 40 cycles $\left(95^{\circ} \mathrm{C}\right.$ for $15 \mathrm{sec}$, $57^{\circ} \mathrm{C}$ for $\left.1 \mathrm{~min}\right)$. To assess the specificity of the amplified product, a melting curve analysis was utilized.

The ribosomal protein S18 (RPS18) gene was used as a reference gene. The PCR reaction was carried out on the Stratagene Mx3005P qPCR System (Agilent Technologies, Germany). The relative expression of the LIAS gene was presented as fold change by the $2^{-\Delta \Delta C T}$ method.

\section{Statistical analysis}

The study parameters showed a non-normally distribution pattern (Shapiro-Wilk test, $\mathrm{p}<0.05$ ), so the quantitative variables were expressed as median and range. The qualitative variables were presented as frequency and percentage. The Kruskal-Wallis $\mathrm{H}$ test and the post hoc test (Dunn's test) were used for the comparison of multiple variables. The JonckheereTerpstra test was utilized to determine the trend of LIAS gene expression with the advancement of disease severity.

Mann- Whitney U test and Chi-squared test were used when appropriate. Receiver operating characteristic (ROC) analysis was created to determine the cutoff value. The association between LIAS gene expression and disease characteristics was performed using the Spearman's correlation coefficient method. The odds ratio was calculated to assess the risk. SPSS 20.0 (Chicago, IL, USA) is the statistical program used in this study and the significance was established at p-value < 0.05 .

\section{RESULTS}

The characteristics of patients and controls are shown in table 1. Among DN patients, 46 patients had microalbuminuria (UACR: $30-300 \mathrm{mg} / \mathrm{gm}$ ) and 14 patients had macroalbuminuria (UACR: $>300 \mathrm{mg} / \mathrm{gm}$ ). 
Table 1: Demographic, clinical and biochemical characteristics of the studied groups.

\begin{tabular}{|c|c|c|c|c|}
\hline Parameter & $\begin{array}{c}\text { Control group } \\
(\text { No. }=60)\end{array}$ & $\begin{array}{l}\text { Diabetes group } \\
\text { (No. = 60) }\end{array}$ & $\begin{array}{l}\text { DN group } \\
(\text { No. }=60)\end{array}$ & $\mathbf{p}$ \\
\hline Age (years) & $42[33.6-52.0]$ & $41.2[32-49.6]$ & $41.6[32-56.3]$ & 0.43 \\
\hline $\begin{array}{l}\text { Sex } \\
\text { Male/ Female }\end{array}$ & $\begin{array}{c}30 / 30 \\
(50 / 50 \%)\end{array}$ & $\begin{array}{c}39 / 21 \\
(65 / 35)\end{array}$ & $\begin{array}{c}38 / 22 \\
(63.3 / 36.7)\end{array}$ & 0.19 \\
\hline Duration of disease \# & --- & $13.6[5.5-17.2]$ & $4.6[1.7-6.4]$ & \\
\hline BMI (Kg/m2) & 25.4 [23.3-29.5] & $25.4[23.3-29.4]$ & $26.6[22.7-31.4]$ & 0.29 \\
\hline Fasting glucose $(\mathrm{mg} / \mathrm{dL})$ & $81.3[74.8-105.0]$ & $121.7[108.3-224.3]^{\mathrm{a}}$ & $132.3[98-313.6]^{\mathbf{a}}$ & $<0.001 *$ \\
\hline HbA1c (\%) & $4.15[3.2-5.0]$ & $6.1[4.9-8.9]^{\mathrm{a}}$ & $7.9[5.5-15.2]^{\mathrm{a}, \mathrm{b}}$ & $<0.001 *$ \\
\hline Cholesterol (mg/dL) & 121.5 [76.4-188.3] & $120.5[88-171.3]$ & $157.7[100.9-289.5]^{\mathbf{a}, \mathbf{b}}$ & $<0.001 *$ \\
\hline Triglyceride (mg/dL) & $82.3[65.3-145.6]$ & $85.1[74-124.2]$ & $98.6[72.4-199.4]^{\mathbf{a}, \mathbf{b}}$ & 0.009 \\
\hline HDL-C (mg/dL) & $43.3[30.4-62.3]$ & $42.1[26.9-55.2]$ & $44.3[29.8-57.4]$ & 0.21 \\
\hline $\mathrm{LDL}-\mathrm{C}(\mathrm{mg} / \mathrm{dL})$ & $60.7[26.2-122.5]$ & $64.4[22.9-114.5]$ & $89[40.5-226.9]^{\mathbf{a}, \mathbf{b}}$ & $<0.001 *$ \\
\hline Serum creatinine $(\mathrm{mg} / \mathrm{dL})$ & $0.65[0.54-1.02]$ & $0.92[0.83-1.15]^{\mathrm{a}}$ & $1.02[0.81-3.2]^{\mathrm{a}}$ & $<0.001 *$ \\
\hline Urinary albumin $(\mathrm{mg} / \mathrm{L})$ & $5.65[3.5-12]$ & $22.3[6.7-32]^{\mathrm{a}}$ & $161.8[66.7-365]^{\text {a,b }}$ & $<0.001 *$ \\
\hline Urinary creatinine $(\mathrm{mg} / \mathrm{dL})$ & 118.8 [55.3-185.3] & $116.3[77.6-133.5]^{\mathrm{a}}$ & $99.7[32-126.3]^{\mathbf{a}, \mathbf{b}}$ & $<0.001^{*}$ \\
\hline UACR (mg/gm) & 5.89 [2.94-10.95] & $20.5[8.5-27]^{\mathrm{a}}$ & $205.8[70.9-500]^{\mathbf{a}, \mathbf{b}}$ & $<0.001 *$ \\
\hline
\end{tabular}

No.: number of subjects; DN: Diabetic nephropathy; BMI: Body mass index; HbAlc: Hemoglobin Alc; HDL-C: high-density lipoprotein cholesterol; LDL-C: Low-density lipoprotein cholesterol; UACR: urinary albumin creatinine ratio; LIAS: Lipoic acid synthetase.

Data are presented as No. (\%) or median [range]

\#: Duration of disease: diabetes in diabetes group and nephropathy in DN group

p: Significance of Kruskal-Wallis $H$ test then post hoc test (Dunn's test):

a: The significance difference in comparison to control group

$b$ : The significance difference in comparison to diabetes group

* Significant

In diabetic and DN patients, the expression of LIAS was significantly lower than controls $(\mathrm{p}<0.001)$. Patients with microalbuminuria had decreased levels in comparison to normoalbuminuria diabetic ones $(p<0.001)$ (Figure 1). Advanced disease severity was associated with lower LIAS gene expression $(p<0.001$, for trend). LIAS gene expression in patients with microalbuminuria and patients with macroalbuminuria was justified. An effect size of 0.87 and a power of 0.8 were detected.

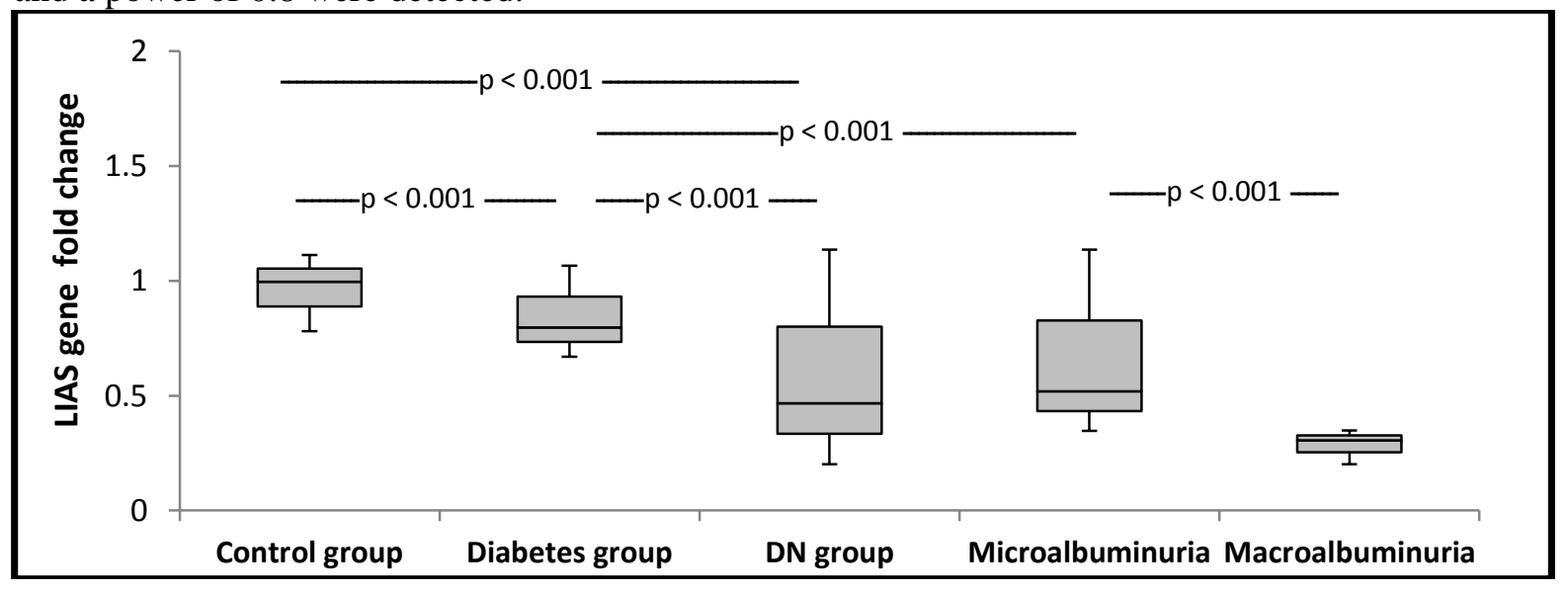

Figure 1: Box and whisker plot of relative LIAS gene expression in healthy controls and different patients groups. Median and range: 1 (0.78-1.11) in controls, 0.8 (0.67-1.07) in diabetes, $0.47(0.2-1.14)$ in DN, 0.52 (0.35-1.14) in microalbuminuria, and 0.31 (0.2-0.35) in macroalbuminuria.

The role of LIAS gene expression in DN detection is illustrated in figure 2. The LIAS gene expression at a cut-off ( $<0.76$ fold change) had $95 \%$ sensitivity, $88.3 \%$ specificity, $89.1 \%$ positive predictive value (PPV), $94.6 \%$ negative predictive value (NPV), and $91.7 \%$ total diagnostic accuracy in the differentiation between DN and diabetes groups (Figure 2a). The performance of the LIAS gene in early detection of DN was assessed.

A ROC curve was created to differentiate patients with microalbuminuria from those with normoalbuminuria (Figure $2 b$ ). At the same cut-off value, LIAS showed $93.5 \%$ sensitivity, $90 \%$ specificity, $87.8 \%$ PPV, $94.7 \%$ NPV, and $91.5 \%$ total diagnostic accuracy. The ROC analysis was justified against the sample size. The ROC AUC of DN diagnosis showed an effect size of 0.74 , and ROC AUC detecting early DN showed 0.72 . The yielded powers were 0.98 and 0.95 , respectively. 

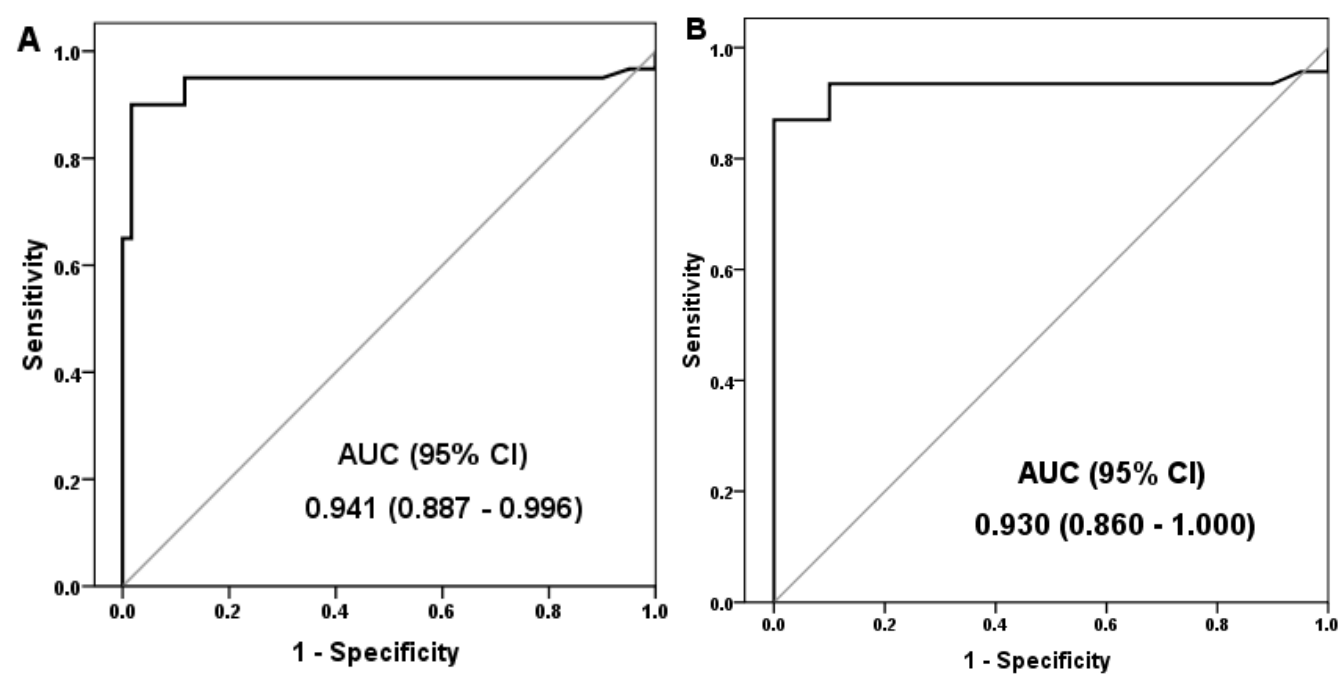

Figure 2: ROC curve for relative LIAS gene expression. (A) Differentiation between DN and diabetes (B) Differentiation between early DN and diabetes

AUC: Area under curve; CI: Confidence interval, DN: Diabetic nephropathy

In table 2, LIAS gene expression in the diabetes group showed significant correlations with disease duration, HbA1c, cholesterol, LDL-C, and serum creatinine. LIAS expression in DN patients had significant correlations with disease duration, HbA1c, serum creatinine, urinary albumin, urinary creatinine, and UACR.

Table 2: Association of relative LIAS gene expression and the clinical and the laboratory data in the studied patients

\begin{tabular}{|l|c|c|c|c|}
\hline \multirow{2}{*}{\multicolumn{1}{c|}{ Parameter }} & \multicolumn{2}{c|}{$\begin{array}{c}\text { Diabetes group } \\
\text { (No. = 60) }\end{array}$} & \multicolumn{2}{c|}{$\begin{array}{c}\text { DN group } \\
\text { (No. =60) }\end{array}$} \\
\cline { 2 - 5 } & r & p & r & p \\
\hline UACR & -0.23 & 0.08 & -0.61 & $<0.001^{*}$ \\
\hline Serum creatinine & -0.47 & $<0.001^{*}$ & -0.47 & $<0.001^{*}$ \\
\hline HbA1c & -0.34 & $0.009^{*}$ & -0.43 & $0.001^{*}$ \\
\hline Duration of disease \# & -0.33 & $0.01^{*}$ & -0.34 & $0.008^{*}$ \\
\hline Urinary creatinine & 0.06 & 0.66 & 0.45 & $<0.001^{*}$ \\
\hline Cholesterol & -0.38 & $0.003^{*}$ & -0.03 & 0.84 \\
\hline LDL-C & -0.37 & $0.004^{*}$ & -0.02 & 0.92 \\
\hline Urinary albumin & -0.24 & 0.07 & -0.33 & $0.01^{*}$ \\
\hline BMI & 0.23 & 0.07 & 0.05 & 0.72 \\
\hline Age & -0.16 & 0.90 & -0.17 & 0.19 \\
\hline Triglycerides & -0.15 & 0.25 & -0.13 & 0.34 \\
\hline Fasting glucose & 0.12 & 0.37 & 0.21 & 0.11 \\
\hline HDL-C & -0.11 & 0.41 & 0.21 & 0.11 \\
\hline Correlation
\end{tabular}

r: Correlation coefficient; DN: Diabetic nephropathy; BMI: Body mass index; HbAlc: Hemoglobin Alc; HDL-C: high-density lipoprotein cholesterol; LDL-C: Low-density lipoprotein cholesterol; UACR: Urinary albumin creatinine ratio.

\# Duration of disease: diabetes in diabetes group and nephropathy in DN group

* Significant

The univariate analysis showed that LIAS gene expression protects significantly from the DN. It had an odds ratio of 0.01 [95\% confidence interval (CI): 0.0010.03 ] $(\mathrm{p}<0.001)$. In multivariate logistic regression analysis, the odds ratio was adjusted for age, sex, duration of the disease, BMI, and HbA1c. LIAS gene expression had an adjusted odds ratio of 0.03 [95\% CI: 0.001-0.02] $(\mathrm{p}<0.001)$.

The logistic regression assessed the ability of laboratory indices to predict the disease severity (controls to diabetic to $\mathrm{DN}$ ). HbA1c, serum creatinine, and LIAS gene expression significantly predicted the disease severity. In a multivariate analysis for the significant variables, the gene expression was only still significantly associated with the disease severity. The low LIAS expression was associated with the diabetic group and DN group in comparison with the control group $(\mathrm{AOR}=6.1,95 \% \mathrm{CI}=1.2-33.3, \mathrm{p}=0.036)$ and (AOR=70.4, 95\% CI=9.3-534, $\mathrm{p}<0.001$ ), respectively. Also, low expression of LIAS increased the risk of DN 
in comparison to diabetic patients, with AOR of 11.5 (95\% CI=3.2- 41.7, $\mathrm{p}<0.001)$.

\section{DISCUSSION}

DN has a high prevalence in type 2 diabetic patients nearly $40 \%$ (27). Deficiency in the endogenous antioxidant results in an inability to compensate for excessive amounts of reactive oxygen species which leads to $\mathrm{DN}^{(28)}$. The understanding of the pathogenesis mechanisms of DN is helping for new predictive, diagnostic, and therapeutic tools to come into the view.

LIAS gene is responsible for lipoic acid synthesis (29). Defective biosynthesis of lipoic acid leads to multiple metabolic abnormalities ${ }^{(30)}$. As far as our knowledge, this study is the first investigation for the expression of the LIAS gene in human diabetic patients.

This study found a reduced relative expression of LIAS gene in diabetes and DN groups. The low expression of LIAS was proportional to the degree of albuminuria. These results support a possible role of the LIAS gene in the development of DN. The LIAS gene expression showed a significantly reduced trend with advanced disease severity. In agreement with previous studies performed in animal models, the LIAS expression was reduced by nearly $30 \%$ in diabetic mice kidney tissues ${ }^{(18)}$. The augmented oxidative stress was revealed as a consequence of LIAS deficiency. This oxidative stress causes DN progress ${ }^{(23)}$. Xu et al. (24) found that the reduction of LIAS gene expression at the mice kidney cortex is associated with DN characteristics severity.

The potential role of LIAS expression as a marker for the diagnosis of DN was assessed. ROC curve analysis showed acceptable performance criteria. LIAS expression was able to differentiate between DN and diabetic patients. Also, LIAS expression can discriminate microalbuminuric patients from those who had normal levels. So, it seems to be a useful marker for early DN detection. The current study revealed that LIAS expression seems to be an independent predictor factor for DN. LIAS expression can predict disease severity.

In diabetic patients, LIAS expression was negatively correlated with dyslipidemia markers except for HDL-C. Similarly, a study demonstrated that LIAS reduction related to the degree of atherosclerosis in diabetic mice ${ }^{(31)}$. In diabetic patients, the lipid-lowering action of lipoic acid was directed mostly on cholesterol and LDL-C ${ }^{(32)}$.

In DN patients, LIAS expression was correlated negatively with serum creatinine, urinary albumin, and UACR. A positive correlation between LIAS and urinary creatinine was detected. These findings may indicate a possible association of LIAS with kidney disease deterioration. The role of lipoic acid in the prevention of early diabetic glomerular injury was previously determined ${ }^{(13)}$. In diabetic and DN patients, LIAS gene expression had indirect correlation with HbA1c. This finding supports the effect of long-term uncontrolled glycemic status on gene expression.
This study revealed that LIAS expression was negatively correlated with diabetes duration. The duration of diabetes influence LIAS under-expression due to the presence of higher levels of oxidant stress markers in prolonged diabetes duration ${ }^{(33)}$.

The prolonged duration of diabetes is linked with its pathologic complications. The duration of diabetes is a confirmed DN risk factor. Clinically, DN progresses over 10-20 years ${ }^{(34)}$. LIAS expression was negatively correlated with DN duration. This suggests that the assessment of LIAS expression may be useful for detecting the early stages of DN and its progression.

As exogenous lipoic acid administration for diabetic patients causes a reduction in fasting glucose and insulin concentrations ${ }^{(35)}$. It improves the diabetic neuropathy symptoms ${ }^{(36)}$. As well as, it prevented the deterioration of diabetic retinopathy ${ }^{(37)}$. The evaluation of lipoic acid administration in DN is recommended.

The main limitation of the current study was the clinical diagnosis of $\mathrm{DN}$, as it was not by the renal biopsy. Further studies are required to establish role of LIAS expression in DN. Also, other studies are needed to detect the reliability of enhancing the endogenous antioxidants as a prevention or treatment modality for DN.

\section{CONCLUSION}

Peripheral blood expression of the LIAS gene was significantly lower in diabetic and DN patients in comparison to controls. The expression of LIAS is negatively correlated with the progress of DN. LIAS gene expression seems to be a promising marker for prediction and early detection of $\mathrm{DN}$ in type 2 diabetic patients.

\section{Disclosure statement:}

The authors report no conflicts of interest.

\section{Funding details:}

This research did not receive any fund.

\section{REFERENCES}

1. Dlamini $Z$, Mbita Z, Mphahlele $L$ (2013): Characterization of the RPL9 and LIAS genes in lung tumors. American Association for Cancer Research, 73(8): 1928.

2. Mantovani G, Maccio A, Madeddu C et al. (2003): Antioxidant agents are effective in lymphocyte progression through cell cycle in advanced cancer patients. J. Mol. Med., 81(10): 664-673.

3. Solmonson A, DeBerardinis R (2018): Lipoic acid metabolism and mitochondrial redox regulation. J Biol Chem., 293(20):7522-7530.

4. Ghibu S, Richard C, Vergely C et al. (2009): Antioxidant properties of an endogenous thiol: Alpha-lipoic acid, useful in the prevention of cardiovascular diseases. J Cardiovasc Pharmacol., 54(5):391-398.

5. Foley R, Collins A (2007): End-stage renal disease in the United States: An update from the United States Renal Data System. J Am Soc Nephrol., 18: 2644-2648. 
6. West I (2000): Radicals and oxidative stress in diabetes. Diabetic Medicine, 17 (10): 171-180.

7. Ha H, Hwang I, Park J et al. (2008): Role of reactive oxygen species in the pathogenesis of diabetic nephropathy. Diabetes Res Clin Pract., 82 (1):42-45.

8. Blum S, Vardi M, Levy N et al. (2010): The effect of vitamin $\mathrm{E}$ supplementation on cardiovascular risk in diabetic individuals with different haptoglobin phenotypes. Atherosclerosis, 211(1):25-27.

9. Papanas N, Ziegler D (2014): Efficacy of $\alpha$-lipoic acid in diabetic neuropathy. Expert Opin Pharmacother., 15(18):2721-2731.

10. Agathos E, Tentolouris A, Eleftheriadou I et al. (2018): Effect of $\alpha$-lipoic acid on symptoms and quality of life in patients with painful diabetic neuropathy. J Int Med Res., 46(5):1779-1790.

11. Bhatti F, Mankhey R, Asico L et al. (2005):

Mechanisms of antioxidant and pro-oxidant effects of $\alpha$ lipoic acid in the diabetic and nondiabetic kidney.

Kidney Int., 67(4):1371-1380.

12. Melhem M, Craven $P$, Liachenko J et al. (2002):

Alpha-lipoic acid attenuates hyperglycemia and prevents glomerular mesangial matrix expansion in diabetes. J Am Soc Nephrol., 13: 108-116.

13. Melhem M, Craven P, Derubertis F (2001): Effects of dietary supplementation of alpha-lipoic acid on early glomerular injury in diabetes mellitus. J Am Soc Nephrol., 12: 124-133.

14. Siu B, Saha J, Smoyer W et al. (2006): Reduction in podocyte density as a pathologic feature in early diabetic nephropathy in rodents: Prevention by lipoic acid treatment. BMC Nephrol., 7: 6.

15. Oksala N, Lappalainen J, Laaksonen D et al. (2007): Alpha-lipoic acid modulates heat shock factor-1 expression in streptozotocin-induced diabetic rat kidney. Antioxid Redox Signal, 9: 497-506.

16. Lee S, Kang JG, Ryu O et al. (2009): Effects of alphalipoic acid on transforming growth factor beta1-p38 mitogen activated protein kinase-fibronectin pathway in diabetic nephropathy. Metabolism, 58: 616-623.

17. Kanter M, Sen S, Donmez S et al. (2010): Protective effects of irbesartan and alpha lipoic acid in STZ-induced diabetic nephropathy in rats. Renal Failure, 32:(4): 498505.

18. Yi X, Nickeleit V, James L et al. (2011): a-Lipoic acid protects diabetic apolipoprotein E-deficient mice from nephropathy. J Diabetes Complications, 25(3): 193-201.

19. Feng B, Yan X-F, Xue J-L et al. (2013): The protective effects of $\alpha$-lipoic acid on kidneys in type 2 diabetic Gotokakisaki rats via reducing oxidative stress. International Journal of Molecular Sciences, 14(4):6746-6756.

20. Ayhan M, Guney E, Atile N et al. (2014): Preventive effects of alpha-Lipoic acid on diabetic nephropathy in a rat model. Acta Medica Mediterranea, 30:1221.

21. Chang J, Lee E, Kim T et al. (2007): Effects of alphalipoic acid on the plasma levels of asymmetric dimethylarginine in diabetic end-stage renal disease patients on hemodialysis: A pilot study. Am J Nephrol., 27: 70-74.
22. Hong Y, Peng J, Cai X et al. (2017): Clinical efficacy of alprostadil combined with $\alpha$-lipoic acid in the treatment of elderly patients with diabetic nephropathy. Open Medicine, 12:323-327.

23. Yi X, Xu L, Hiller S et al. (2012): Reduced expression of lipoic acid synthase accelerates diabetic nephropathy. J Am Soc Nephrol., 23(1):103-111.

24. Xu L, Hiller S, Simington S et al. (2016): Influence of different levels of lipoic acid synthase gene expression on diabetic nephropathy. PLoS ONE, 11(10): 0163208.

25. American Diabetes Association (2010): Diagnosis and classification of diabetes mellitus, Diabetes care, 33 (1) 62-69.

26. American Diabetes Association (2004): Nephropathy in diabetes (Position Statement). Diabetes Care, 27(1) 7983.

27. Aldukhayel A (2017): Prevalence of diabetic nephropathy among Type 2 diabetic patients in some of the Arab countries. Int J Health Sci (Qassim), 11(1):1-4.

28. Padmalayam I, Hasham S, Saxena U et al. (2009): Lipoic acid synthase (LASY): a novel role in inflammation, mitochondrial function, and insulin resistance. Diabetes, 58(3):600-608.

29. Mphahlele L, Dlamini Z (2006): Molecular evaluation of lipoic acid synthetase gene in lung cancer. Proc Amer Assoc Cancer Res., 47 (8):1239.

30. Baker P, Friederich M, Swanson M et al. (2014): Variant non ketotic hyperglycinemia is caused by mutations in LIAS, BOLA3 and the novel gene GLRX5. Brain, 137(2):366-379.

31. Yi X, Xu L, Hiller S et al. (2012): Reduced alpha-lipoic acid synthase gene expression exacerbates atherosclerosis in diabetic apolipoprotein e-deficient mice. Atherosclerosis, 223(1):137-143.

32. Haghighatdoost F, Hariri M (2019): Does alpha-lipoic acid affect lipid profile? A meta-analysis and systematic review on randomized controlled trials. Eur J Pharmacol., 847:1-10.

33. Hsu W, Tsai L, Lin S et al. (2006): Effects of diabetes duration and glycemic control on free radicals in children with type 1 diabetes mellitus. Annals of clinical and laboratory science, 36:174-178.

34. Sulaiman M (2019): Diabetic nephropathy: recent advances in pathophysiology and challenges in dietary management. Diabetol. Metab. Syndr., 11: 7.

35. Akbari M, Ostadmohammadi V, Lankarani $\mathrm{K}$ et al. (2018): The effects of alpha-lipoic acid supplementation on glucose control and lipid profiles among patients with metabolic diseases: A systematic review and metaanalysis of randomized controlled trials. Metabolism, 87:56-69.

36. Ziegler D, Low P, Litchy W et al. (2011): Efficacy and safety of antioxidant treatment with alpha-lipoic acid over 4 years in diabetic polyneuropathy: the NATHAN 1 trial. Diabetes Care, 34(9):2054-2060.

37. Gebka A, Serkies-Minuth E, Raczynska D (2014): Effect of the administration of alpha-lipoic acid on contrast sensitivity in patients with type 1 and type 2 diabetes. Mediators Inflamm., 2014:131538. 